\title{
Next Generation Sequencing for Microbial Analysis to Select Prophylactic Antibiotic Selection before Urologic Stone Surgery: A Culture Change
}

\author{
Rishi Das ${ }^{*}$, Tim Tseng1 ${ }^{*}$, Meghan I. Short ${ }^{2}$, Kelly Reveles ${ }^{3}$, Allison Wheeler ${ }^{1}$, Robert Hudson1, \\ Bernard Fongang ${ }^{3}$, Michael A. Liss ${ }^{1,3 \#}$
}

${ }^{1}$ Department of Urology, University of Texas Health San Antonio, San Antonio, USA

${ }^{2}$ Glenn Biggs Institute for Alzheimer's and Neurodegenerative Diseases, San Antonio, Texas, USA

${ }^{3}$ The University of Texas at Austin-College of Pharmacy (PHR), Austin, USA

Email: "liss@uthscsa.edu

How to cite this paper: Das, R., Tseng, T., Short, M.I., Reveles, K., Wheeler, A., Hudson, R., Fongang, B. and Liss, M.A. (2021) Next Generation Sequencing for Microbial Analysis to Select Prophylactic Antibiotic Selection before Urologic Stone Surgery: A Culture Change. Open Journal of Urology, 11, 289-304.

https://doi.org/10.4236/oju.2021.117027

Received: April 6, 2021

Accepted: July 23, 2021

Published: July 26, 2021

Copyright $\odot 2021$ by author(s) and Scientific Research Publishing Inc. This work is licensed under the Creative Commons Attribution International License (CC BY 4.0).

http://creativecommons.org/licenses/by/4.0/

\begin{abstract}
Background: This paper aims to determine if the combination of polymerase chain reaction (PCR) and next-generation sequencing (NGS) could identify bacteria in culture-negative urine that would alter prophylaxis management. Methods: We sent approximately $5-10 \mathrm{~mL}$ of a preoperative urine sample to MicrogenDx for PCR/NGS analysis performed after surgery (blind to the surgeon). The physician prescribed standard of care antibiotic prophylaxis. Cases modeling the hospital course of 3 random patients were reviewed by eight urologists after surgery to determine if NGS results would change their prophylaxis regimen. An infectious disease pharmacist reviewed the cases and provided the "ideal" regimen. Results: Urine cultures identified bacteria in $11 \%(2 / 18)$ of cases. Culture speciation results were consistent with NGS results. NGS detected a dominant bacteria in 56\% (10/18) of negative cultures and targetable bacteria in all samples. There was a $15 \%(3 / 20)$ infection rate. In both cases, NGS results suggest inadequate prophylaxis. In response to the case scenarios, $100 \%, 88 \%$, and $88 \%$ of the urologists reported they would change prophylaxis with NGS results. During a case scenario, physicians would tend to overprescribe antibiotics given PCR/NGS data for prophylaxis selection. Conclusion: NGS identifies a targetable bacterium in up to $80 \%$ of negative urine cultures before urologic stone surgery. Responses to case scenarios indicate that physicians would change management based on NGS results. Inter-professional (urologic and pharmacy) antibiotic selection with PCR/16S DNA testing may be helpful to improve antibiotic stewardship.
\end{abstract}




\section{Keywords}

Health-Care Associated Infection, Urinary Tract Infection, Infection Prevention, Surgical Site Infection

\section{Introduction}

Ureteroscopy for urinary tract urolithiasis is a standard procedure with an estimated 9200 cases in the US per year [1]. Despite antibiotic prophylaxis, the incidence of significant infections after flexible ureteroscopic (URS) lithotripsy is on the rise [2]. To prevent infections, the standard of care is to obtain a negative urine culture before surgery. However, with the next-generation sequencing, we are now able to understand that the urine is not sterile and that pathogenic bacteria may be still present, undetected by urine culture.

One of the challenges when choosing a prophylactic agent is that preoperative urine cultures often show no growth for patients who later develop SIRS [3] [4] [5]. Singh et al. found no significant association between pelvic urine cultures or stone cultures and the occurrence of SIRS [6]. We hypothesize the stone or previously placed stent may allow bacteria to form biofilms containing a small number of essential bacteria not detected by standard cultures.

Next-generation sequencing (NGS) poses an alternative to the traditional culture model by using high-throughput sequencing of rapid PCR for resistance genes combined with 16S rRNA (a type of NGS) to detect specific bacterial strains and has been used for the detection of urinary tract infections [7]. With its improved sensitivity, NGS (PCR/16s rRNA) with resistance genes could be used to guide antibiotic therapy. In this study, we pilot this novel approach quantitatively to evaluate the NGS platform's ability to identify bacteria in culture-negative urine that would alter the choice of antibiotic prophylaxis for patients undergoing urologic stone procedures. We also evaluate this approach qualitatively to determine its usefulness to urologic surgeons to inform clinical trial design.

\section{Materials and Methods}

\subsection{Population}

After IRB approval (HSC20050234H), subjects were recruited from urology clinics at their preoperative appointment before their planned ureteroscopy (URS) surgery for urinary stone. We did not exclude patients with ureteral stents, including all patients undergoing percutaneous nephrolithotomy (PCNL) or URS within the next two weeks. We informed patients that the results were purely for research purposes, and no analysis performed until after surgery. We also described we would not provide additional information to their physician that could alter antibiotics. 


\subsection{Next-Generation Sequencing}

We collected whole urine (approximately $30-50 \mathrm{~mL}$ ) utilizing special vaccutanors supplied by MicrogenDx. We sent around $5-10 \mathrm{~mL}$ of urine taken for culture to MicroGen Diagnostics, a CAP-accredited and CLIA licensed clinical diagnostic lab, for analysis. MicrogenDx performed rapid PCR for common resistance genes in 21 subjects before URS. Each initial target bacterial or fungi DNA, whose concentration was measured to obtain an initial concentration (ng/uL), was diluted to obtain a six to eight-fold serial dilution series and run on the quantitative PCR (qPCR) panel assay on the Roche LightCycler 480 II instrument. We sequenced the V1-V2 hypervariable bacterial regions with 16S RNA using Ion Torrent (Ion Torrent PGM). Physicians did not obtain results before surgery. The physician proceeded with surgical management and standard of care antibacterial prophylaxis.

\subsection{Data Collection}

We recorded data from physician notes and medical record review regarding the details of patient history, urologic stone procedure, postoperative course, and infection outcomes. We recorded all speciation results from the standard of care urine cultures. We then compared these results to speciation results in the PCR/ NGS (16s rRNA).

\subsection{Case Study Creation}

We perform a qualitative review in the form of case scenarios to determine if NGS would indeed alter antibiotic prophylaxis. Choosing from the cases enrolled, we randomly selected three cases to be reviewed by eight board-certified Urologists. One infectious disease pharmacists also reviewed the case reports and recommended the "ideal" antibiotic regimen for each case. The cases are highlighted in Supplemental Figures S1-S3 representing each case scenario to include:

Case 1: Standard culture No Growth, PCR/16s showed primary bacteria with Enterococcus (82\%) and secondary Staphylococcus (17\%) with resistance genes for macrolide and aminoglycoside.

Case 2: Standard culture showed no growth; PCR/16s showed primary bacteria of Enterococcus (99\%) with no resistance genes.

Case 3: Standard culture showed "normal flora," PCR/16s showed Citrobacter (79\%), and E. coli (12\%) with resistance genes of methicillin, beta-lactam, quinolone, and macrolide.

The questions included:

1) Based on PCR/16s results, would you change your antibiotic?

2) Choose from your usual antibiotic choice, what antibiotic would you choose?

3) Exit survey questions:

a) Would this test improve my confidence to prevent infection?

b) Would I use this test again?

c) Would I recommend this test to other urologists? 
d) How important do you think this test would be in urologic practice?

e) I have concerns about using this technology for antibiotic prophylaxis selection?

\section{Results}

\subsection{Patient Characteristics}

Participants had a median age of 59 (interquartile range: 37 - 70), were predominantly white (80\%), and non-Hispanic (60\%). They had a median BMI of 30.8 (interquartile range: 24.8 - 34.1). Most patients $(14,70 \%)$ were not diabetic. Most patients $(12,60 \%)$ did not have any allergies to antibiotics. $5(25 \%)$ patients had indwelling stents when urine was collected, and 5 (25\%) patients had percutaneous nephrostomy tubes (Table 1).

\subsection{Surgical Methods}

A variety of urologic stone procedures were represented in this cohort, with cases of PCNL and URS, laser lithotripsy, and basket retrieval, with and without

Table 1. Patient characteristics.

\begin{tabular}{|c|c|}
\hline Characteristics $\mathrm{n},(\%)$ & Total patients $(n=20)$ \\
\hline \multicolumn{2}{|l|}{ Gender } \\
\hline Men & $11(55 \%)$ \\
\hline Women & $9(45 \%)$ \\
\hline Age years, (IQR) & $59(37-70)$ \\
\hline \multicolumn{2}{|l|}{ Race } \\
\hline White & $16(80 \%)$ \\
\hline Unknown & $3(15 \%)$ \\
\hline Black & $1(5 \%)$ \\
\hline \multicolumn{2}{|l|}{ Ethnicity } \\
\hline Non-Hispanic & $12(60 \%)$ \\
\hline Hispanic & $6(30 \%)$ \\
\hline Unknown & $2(10 \%)$ \\
\hline Body Mass Index (IQR) & $30.8(24.8-34.1)$ \\
\hline \multicolumn{2}{|l|}{ Diabetes status } \\
\hline Non-Diabetic & $14(70 \%)$ \\
\hline Diabetic & $6(30 \%)$ \\
\hline \multicolumn{2}{|l|}{ History of UTIs } \\
\hline No & $13(65 \%)$ \\
\hline Yes & $7(35 \%)$ \\
\hline \multicolumn{2}{|l|}{ Stent in place on presentation } \\
\hline No & $15(75 \%)$ \\
\hline Yes & $5(25 \%)$ \\
\hline \multicolumn{2}{|c|}{ Catheter in place on presentation } \\
\hline No & $15(75 \%)$ \\
\hline Yes & $5(25 \%)$ \\
\hline
\end{tabular}

Abbreviations: IQR: interquartile range; URS: ureteroscopy. 
nephrostomy tube/ureteral stent placement. There was 1 case of a TURP in addition to URS, and 1 case of an added endopyelotomy (Table 2).

\subsection{Bacterial Identification and Antibiotic Resistance}

Of the 20 cases, only 2 SOC cultures had speciation results and 16s rRNA analysis identified the exact bacteria colonized on both accounts (Figure 1). One patient had grown two bacterial species on culture, and NGS identified both species. In the 10 cases where SOC cultures resulted in no growth, NGS was able to identify targetable bacteria. In only 2 of those 10 cases with no growth, the dominant species detected by NGS (Citrobacter and Lactobacillus) was unlikely to cause postoperative infections. The other dominant species identified in these cases were E. coli and $S$. epidermidis, respectively. In nearly $80 \%$ of negative cultures, NGS provided dominant speciation data to consider when choosing antimicrobial prophylaxis before urologic stone procedures (Figure 2).

\subsection{Cases of Infection}

Of the 18 patients who underwent their procedure, 2 cases (11\%) developed infections postoperatively. In the first infection case, the patient did not supply a urine culture before URS. The surgeon chose cefazolin for antibiotic prophylaxis. NGS detected a high bacterial load (>107): 53\% Morganella morganii and $45 \%$ E. coli. NGS was also able to identify resistance genes against beta-lactams and fluoroquinolones. Based on this data, we would recommend trimethoprimsulfamethoxazole or a $3^{\text {rd }}$ generation cephalosporin rather than a $1^{\text {st }}$ generation cephalosporin. In the second case of infection, three or more organisms present, each higher than $10,000 \mathrm{cu} / \mathrm{mL}$, per SOC UC results. The surgeon chose cefazolin and piperacillin/tazobactam for prophylaxis. NGS detected Citrobacter (64\%), Veillonella atypica (27\%), and resistance genes to methicillin, beta-lactams, macrolides, and aminoglycosides. NGS also detected Candida albicans in its fungal

Table 2. Surgical methods.

\begin{tabular}{cc}
\hline Surgical procedure & Total Patients ( $\mathbf{n}=\mathbf{2 0})$ \\
\hline U PCNL with nephrostomy placement & $3(15 \%)$ \\
B PCNL with nephrostomy placement & $1(5 \%)$ \\
U PCNL & $1(5 \%)$ \\
U URS, LL with stent placement & $5(25 \%)$ \\
B URS, LL with stent placement & $4(20 \%)$ \\
B URS, LL with stent placement & $1(5 \%)$ \\
B URS, LL and unilateral Basket retrieval, bilat stent placement & $1(5 \%)$ \\
U URS, Basket retrieval, TURP & $1(5 \%)$ \\
U URS, endopyelotomy with stent placement & $1(5 \%)$ \\
Surgery cancelled & $2(10 \%)$ \\
\hline
\end{tabular}

Abbreviations: B: bilateral, U: unilateral, LL: laser lithotripsy, URS: ureteroscopy, TURP: transurethral resection of prostate. 


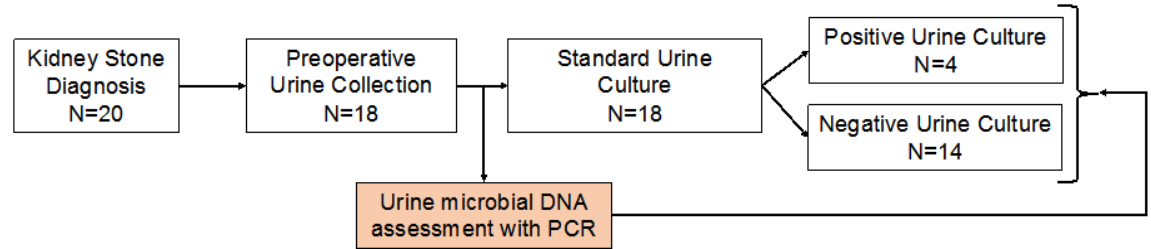

We display our enrollment of 20 subjects through the study. The urine was split between culture and next-generation sequencing with PCR of plasmid genes. We then compared the results of the PCR to the culture specimen as ground truth.

Figure 1. Consort diagram.

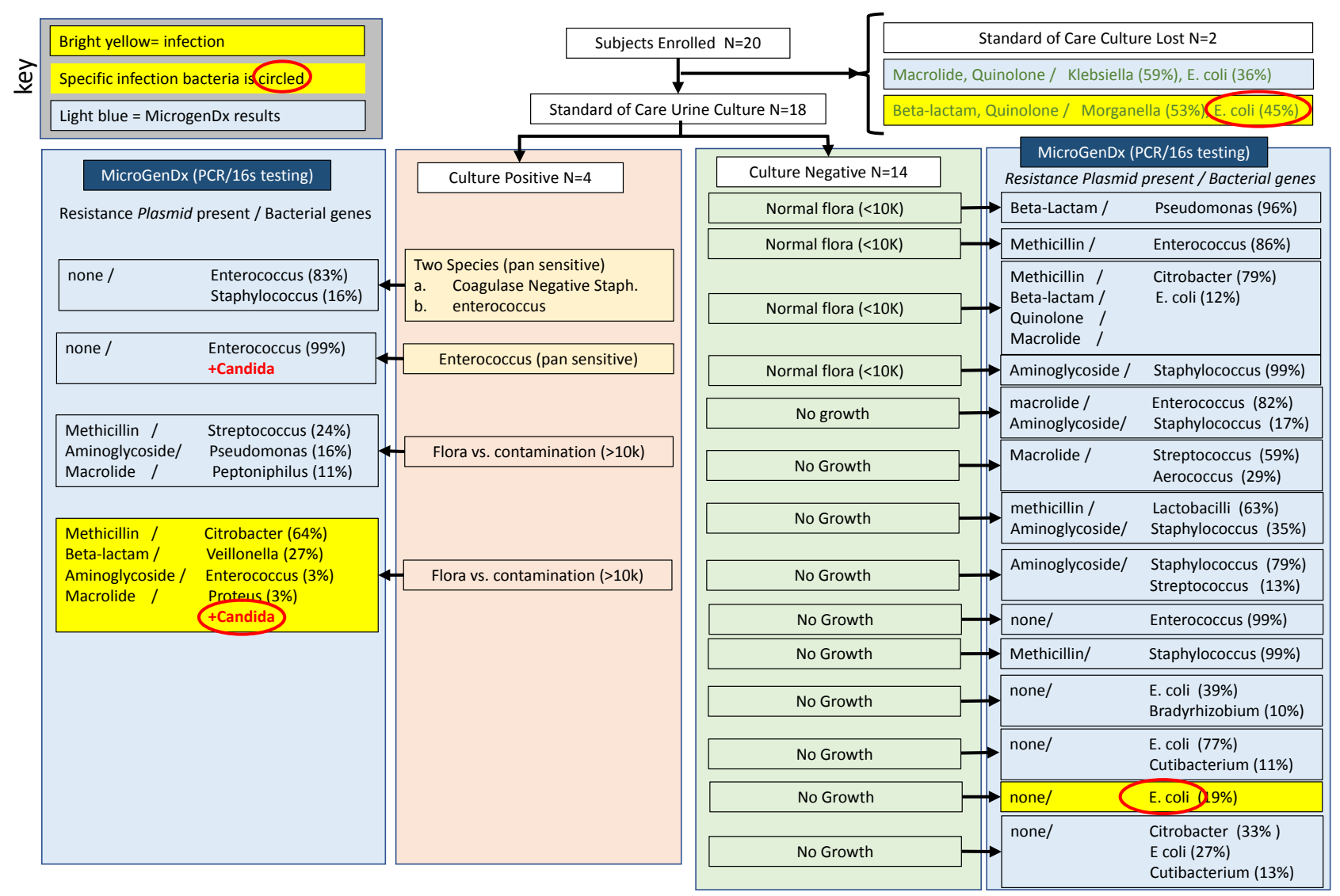

We display a diagram of urine culture and corresponding PCR results from subjects On the left are the positive urine cultures noting alignment between genetic and phenotypic results. Of note, Candida is not routinely cultured before urologic stone procedures. On the right are all of the negative urine culture results and their associated NGS/PCR results. Bright yellow represents those that had postoperative clinical infection with a red circle to indicate the causative organism. The orange squares were those subjects selected for the case reports in the supplemental figures.

Figure 2. Results diagram.

screen as a standard part of the test. The patient developed funguria postoperatively with Candida albicans, indicating that NGS may be specific enough to recommend antifungal prophylaxis appropriately. These findings would be helpful in PCNL patient's that have had multiple antibiotics with high level of suspicion for candida

\subsection{Case Scenario Data}

Eight urologists responded to the three case scenarios, and in each of the 3 cases, $100 \%, 88 \%$, and $88 \%$ of the physicians would have changed their prophylaxis 
management given NGS results (Supplemental Figures S1-S3). The ideal antibiotic of choice based on the ID Pharmacist recommendations was oral Bactrim for all cases, and only $50 \%, 0 \%$, and $0 \%$ selected this option. In contrast, $38 \%$, $25 \%$, and $63 \%$ chose to escalate the antibiotic to vancomycin, Zosyn, or Amikacin in conjunction with a second antibiotic. Overall, on exit survey, urologists reported that it is moderately important $(n=2,25 \%)$, important $(n=4,50 \%)$, or very important $(\mathrm{n}=2,25 \%)$ to test this technology in this context, while $38 \%$ have concerns about using this technology for antibiotic prophylaxis related primarily to implementation.

\section{Discussion}

We report several findings from our pilot study that include: NGS may provide actionable information above standard urine culture, urologists find the information useful, and concern for antibiotic escalation may benefit from interprofessional collaboration with pharmacists to select preoperative antibiotics in the context of PCR/NGS testing. Testing NGS is a practical extension to standard urinary culture in that the specimen can be split and sent for culture and NGS. Results of NGS are available in 48 hours to allow for time for preoperative antibiotic selection well before surgery.

In our first question, we address the usefulness of a PCR/NGS based urine profile before URS in this pilot study. We found that NGS detects targetable bacteria and fungi in culture-negative urine and propose utilizing this data in a prospective trial to use NGS to determine preoperative antibiotic prophylaxis. NGS may be more effective and specific than those predicated upon SOC preoperative urine cultures mostly negative before surgery. For example, we identified several culture-negative patients with dominant bacterial types that may influence a physician to prescribe a $1^{\text {st }}$ generation cephalosporin compared to a gram-negative dominant group that may need a fluoroquinolone or $3^{\text {rd }}$ generation cephalosporin. Importantly, urologists do not typically send a urine specimen specifically for fungi (candida sp.). The PCR/NGS test readily makes this information available, and in one patient that was positive did have sepsis caused by Candida, which was later cultured from the patient's blood. Many studies have reported the low sensitivity of preoperative urine cultures for predicting infectious complications of urologic stone procedures [3] [4] [5] [8]. Eswara et al. (2013) compared sensitivities among preoperative and perioperative pelvic urine and stone cultures for pathogen detection, reporting that of the patients who develop urosepsis, $0 \%$ had positive midstream preoperative urine cultures, while $73 \%$ had positive stone cultures. [9] However, they report a 64\% concordance rate in urosepsis patients between stone cultures and readmission cultures, indicating that stone cultures did not always appropriately guide antibiotic selection in up to $36 \%$ of cases. Moreover, an antibiotic selection from stone culture rarely is provided in a timely fashion to alter postoperative antibiotics, and many of our patients go home the same day of the procedure. Our study, while small, 
found that NGS was able to detect pathogens and provide alternate antimicrobial prophylaxis, especially in those who did have an infection.

Our findings are consistent with those of Long et al. (2016) and Grumaz et al. (2016), who reported that NGS is more sensitive than blood cultures for detecting pathogens in ICU patients Notably, their studies observe the clinical utility of NGS to guide therapy in a high-risk population of ICU patients [10] [11]. Many studies have reported the increased sensitivity of NGS when compared to cultures, but further research is needed to establish cases where the improved sensitivity delivers cost-effective, clinically applicable data. The results of this study suggest that NGS may improve the standard of care in patients undergoing invasive urologic stone procedures.

Infectious complications are the most common cause of death following urologic stone procedures and present a sizeable economic drain on the American healthcare system [1] [12]. Other studies have found that up to $17 \%$ of urosepsis cases follow urologic interventions [13] [14] [15] [16] [17]. Koras et al. (2014) observed signs of SIRS in up to $27 \%$ of patients who underwent PCNL, $7.6 \%$ of which were diagnosed with sepsis [13]. Given the mortality rates and economic costs of urosepsis, SOC practices must be optimized to reduce the risk of infectious complications following urologic procedures.

Our qualitative questions address the usefulness of PCR/NGS testing to urologists before URS. We identified that $88 \%$ - 100\% of urologists reported that they would have changed their prophylactic regimen based on NGS results in the post case survey. However, during the cases, most urologists did not choose the ideal regimen recommended by infectious disease pharmacists and in many escalated antibiotic use to either more antibiotics or broader spectrum. The findings of this study suggest that interdisciplinary collaboration between physicians and pharmacists may prevent excessive use of aggressive therapy when utilizing highly sensitive pathogen detection modalities, such as NGS.

We have several limitations to our study. The sample size limits the power of this study; however, this is a pilot study to inform a larger clinical trial and is informative for planning. We decided to publish these results due to the novelty of the research and its potential to change the preoperative antibiotic selection for a large number of patients. The increased sensitivity of NGS compared to SOC urine cultures reported herein was observed in a cohort of 20 patients. However, $88 \%-100 \%$ of urologists surveyed stated that NGS with PCR for resistance genes would change their management in the 3 case scenarios we prepared, suggesting that physicians would use NGS in cases where it detected bacteria not found on preoperative cultures. More extensive studies are required to conclude that NGS can detect targetable microbes in culture-negative urine. Another limitation of this study is the lack of direct comparisons to perioperative cultures. However, as these perioperative cultures cannot guide antimicrobial prophylaxis, a direct comparison between preoperative studies was used. Lastly, this was a repository study using data from chart review. We did not call patients to follow 
up to confirm the postoperative course. We did not perform a full cost analysis and the current cost of the specific PCR method in this study through MicroGenDx is $\$ 199.00$ US. Urine testing is variable depending on if an analysis was sent first and if the culture is positive then species detection and resistance profiling is performed (average $\$ 30$ - $\$ 80$ ).

\section{Conclusion}

Infectious complications are the most common cause of death following urologic stone procedures. This study found that NGS can identify a targetable bacterium in up to $80 \%$ of negative urine cultures before urologic stone surgery. Responses to case scenarios indicate that physicians would change management based on NGS results. Using this data we have initiated a clinical trial using NGS to augment antibiotic section in urine culture negative patients prior to ureteroscopy for stone surgery (NCT04404855).

\section{Conflicts of Interest}

The authors declare no conflicts of interest regarding the publication of this paper.

\section{References}

[1] Raheem, O.A., Khandwala, Y.S., Sur, R.L., Ghani, K.R. and Denstedt, J.D. (2017) Burden of Urolithiasis: Trends in Prevalence, Treatments, and Costs. European Urology Focus, 3, 18-26. https://doi.org/10.1016/j.euf.2017.04.001

[2] Zhong, W., Leto, G., Wang, L. and Zeng, G. (2015) Systemic Inflammatory Response Syndrome after Flexible Ureteroscopic Lithotripsy: A Study of Risk Factors. Journal of Endourology, 29, 25-28. https://doi.org/10.1089/end.2014.0409

[3] Korets, R., Graversen, J.A., Kates, M., Mues, A.C. and Gupta, M. (2011) Post-Percutaneous Nephrolithotomy Systemic Inflammatory Response: A Prospective Analysis of Preoperative Urine, Renal Pelvic Urine and Stone Cultures. The Journal of Urology, 186, 1899-1903. https://doi.org/10.1016/j.juro.2011.06.064

[4] Mariappan, P., Smith, G., Bariol, S.V., Moussa, S.A. and Tolley, D.A. (2005) Stone and Pelvic Urine Culture and Sensitivity Are Better than Bladder Urine as Predictors of Urosepsis Following Percutaneous Nephrolithotomy: A Prospective Clinical Study. The Journal of Urology, 173, 1610-1614. https://doi.org/10.1097/01.ju.0000154350.78826.96

[5] Sohn, D.W., Kim, S.W., Hong, C.G., Yoon, B.I., Ha, U.S. and Cho, Y.H. (2013) Risk Factors of Infectious Complication after Ureteroscopic Procedures of the Upper Urinary Tract. The Journal of Infection and Chemotherapy, 19, 1102-1108. https://doi.org/10.1007/s10156-013-0632-7

[6] Singh, I., Shah, S., Gupta, S. and Singh, N.P. (2019) Efficacy of Intraoperative Renal Stone Culture in Predicting Postpercutaneous Nephrolithotomy Urosepsis/Systemic Inflammatory Response Syndrome: A Prospective Analytical Study with Review of Literature. Journal of Endourology, 33, 84-92.

https://doi.org/10.1089/end.2018.0842

[7] Mouraviev, V. and McDonald, M. (2018) An Implementation of Next Generation Sequencing for Prevention and Diagnosis of Urinary Tract Infection in Urology. 
The Canadian Journal of Urology, 25, 9349-9356.

[8] Senocak, C., Ozcan, C., Sahin, T., Yilmaz, G., Ozyuvali, E., Sarikaya, S., et al. (2018) Risk Factors of Infectious Complications after Flexible Uretero-Renoscopy with Laser Lithotripsy. Urology Journal, 15, 158-163.

[9] Eswara, J.R., Shariftabrizi, A. and Sacco, D. (2013) Positive Stone Culture Is Associated with a Higher Rate of Sepsis after Endourological Procedures. Urolithiasis, 41, 411-414. https://doi.org/10.1007/s00240-013-0581-8

[10] Long, Y., Zhang, Y., Gong, Y., Sun, R., Su, L., Lin, X., et al. (2016) Diagnosis of Sepsis with Cell-Free DNA by Next-Generation Sequencing Technology in ICU Patients. Archives of Medical Research, 47, 365-371. https://doi.org/10.1016/j.arcmed.2016.08.004

[11] Grumaz, S., Stevens, P., Grumaz, C., Decker, S.O., Weigand, M.A., Hofer, S., et al. (2016) Next-Generation Sequencing Diagnostics of Bacteremia in Septic Patients. Genome Medicine, 8, 73. https://doi.org/10.1186/s13073-016-0326-8

[12] Angus, D.C., Linde-Zwirble, W.T., Lidicker, J., Clermont, G., Carcillo, J. and Pinsky, M.R. (2001) Epidemiology of Severe Sepsis in the United States: Analysis of Incidence, Outcome, and Associated Costs of Care. Critical Care Medicine, 29, 1303-1310. https://doi.org/10.1097/00003246-200107000-00002

[13] Koras, O., Bozkurt, I.H., Yonguc, T., Degirmenci, T., Arslan, B., Gunlusoy, B., et al. (2015) Risk Factors for Postoperative Infectious Complications Following Percutaneous Nephrolithotomy: A Prospective Clinical Study. Urolithiasis, 43, 55-60. https://doi.org/10.1007/s00240-014-0730-8

[14] Kreydin, E.I. and Eisner, B.H. (2013) Risk Factors for Sepsis after Percutaneous Renal Stone Surgery. Nature Reviews Urology, 10, 598-605. https://doi.org/10.1038/nrurol.2013.183

[15] Rivera, M., Viers, B., Cockerill, P., Agarwal, D., Mehta, R. and Krambeck, A. (2016) Pre- and Postoperative Predictors of Infection-Related Complications in Patients Undergoing Percutaneous Nephrolithotomy. Journal of Endourology, 30, 982-986. https://doi.org/10.1089/end.2016.0191

[16] Scotland, K.B. and Lange, D. (2018) Prevention and Management of Urosepsis Triggered by Ureteroscopy. Research and Reports in Urology, 10, 43-49. https://doi.org/10.2147/RRU.S128071

[17] Wagenlehner, F.M., Pilatz, A., Naber, K.G. and Weidner, W. (2008) Therapeutic Challenges of Urosepsis. European Journal of Clinical Investigation, 38, 45-49. https://doi.org/10.1111/j.1365-2362.2008.02008.x 


\section{Supplemental Figures}

Case Scenario 1

A man in his late 50's presents with nephrolithiasis and blood in the urine. He is allergic to penicllins. The patient is planned to undergo bilateral ureteroscopy. SOC urine culture result showed no growth.

What would you select as your antibiotic for surgery prophylaxis?

Based on Microgen results:

1. Would you change your antibiotic?

2. What would you select?

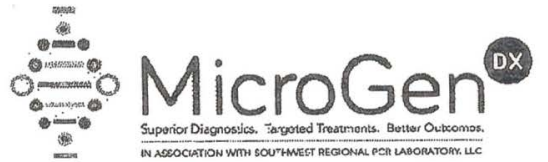

4321 Marsha Sharp Fwy Door 2, Lubbock, TX 79407

Case Scenario 1

\section{Next Generation Sequencing Results}

MicroGen Diagnostics' comprehensive testing (patent pending) is a relative quantitative universal test for bacteria/fungi. DNA sequencing methods are MicroGen Diagnostics' complive used to identify the microorganisms' genetic signatures and the estim
are screened for and the most predominant populations are reported.

\begin{tabular}{|c|c|c|c|}
\hline Rapid Screening (PCR Results) & Amount per $\mathrm{mL}$ & \multicolumn{2}{|c|}{$\begin{array}{l}\text { Comprehensive Identification } \\
\text { (Sequencing Results) }\end{array}$} \\
\hline $\begin{array}{l}\text { Bacterial Load (Medium) } \\
\text { Enterococcus faecal is }\end{array}$ & $\frac{10^{5}-10^{7}}{2.57 \times 10^{5}}$ & Detected Bacteria: & \\
\hline Enterococcus faecalis & & Enterococcus faecalis & $82 \%$ \\
\hline Klebsiella pneumoniae & Not Detected & Staphylococcus epidermidis & $11 \%$ \\
\hline Streptococcus agalactiae & Not Detected & Staphylococcus lugdunensis & $6 \%$ \\
\hline Pseudomonas aeruginosa & Not Detected & & \\
\hline Staphylococcus aureus & Not Detected & NO FUNGAL SPECIES DETECTED & \\
\hline Proteus mirabilis & Not Detected & & \\
\hline Escherichia coli & Not Detected & & \\
\hline Mobiluncus curtisii & Not Detected & & \\
\hline Mobiluncus mulieris & Not Detected & & \\
\hline Gardnerella vaginalis & Not Detected & & \\
\hline Ureaplasma urealyticum & Not Detected & & \\
\hline Ureaplasma parvum & Not Detected & & \\
\hline Staphylococcus saprophyticus & Not Detected & & \\
\hline Prevotella bivia & Not Detected & & \\
\hline Mycoplasma hominis & Not Detected & & \\
\hline Lactobacillus gasseri & Not Detected & & \\
\hline Lactobacillus crispatus/acidophilus & Not Detected & & \\
\hline Resistance Genes Detected & & & \\
\hline Macrolide & & & \\
\hline Aminoglycoside & & & \\
\hline Resistance Genes Not Detected & & & \\
\hline Vancomycin & & & \\
\hline Methicillin & & & \\
\hline Beta-lactam & & & \\
\hline Carbapenem & & & \\
\hline Tetracycline & & & \\
\hline Quinolone & & & \\
\hline
\end{tabular}

Complete Antibiotic Analysis [Next Page(s)]

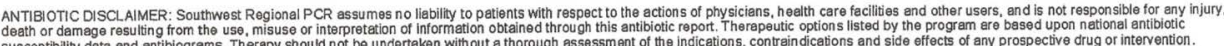

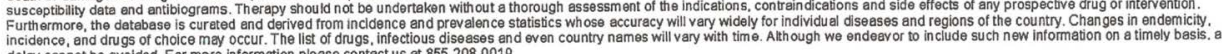

delay cennot po avo 


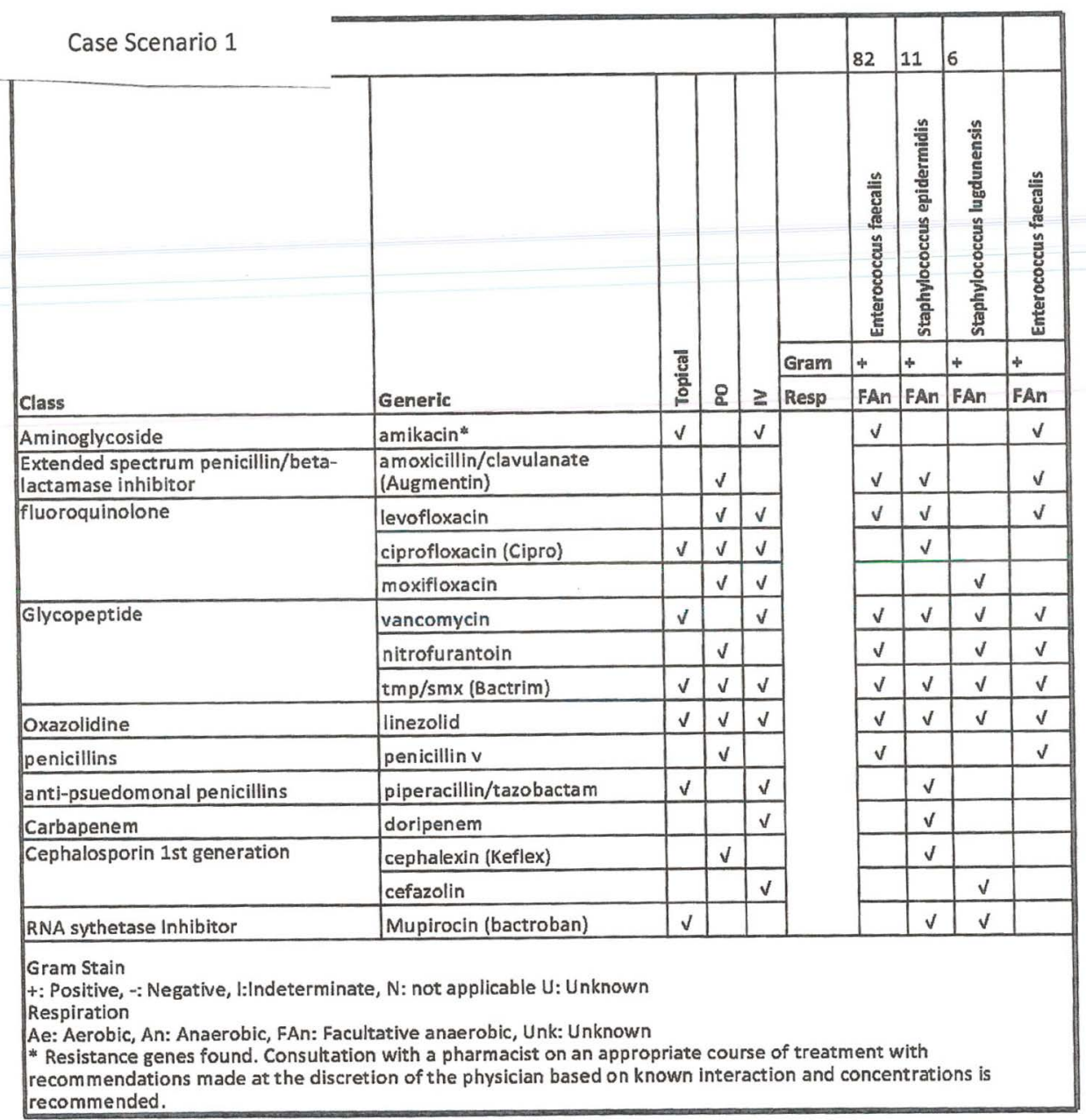

Supplemental Figure S1. Case scenario 1 with microgen. 
Case Scenario 2

A man in his mid 30's presents with nephrolithiasis and a right ureteral calculus. He has no known allergies to antibiotics. The patient is planned to undergo bilateral ureteroscopy. SOC urine culture result showed no growth.

What would you select as your antibiotic for surgery prophylaxis?

Based on Microgen results:

1. Would you change your antibiotic?

2. What would you select?

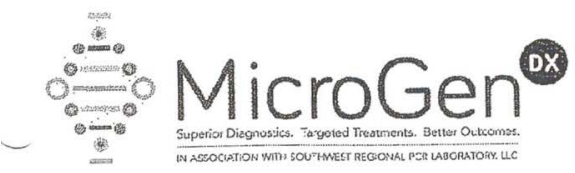

4321 Marsha Sharp Fwy Door 2, Lubbock, TX 79407

\section{Case scenario 2}

\section{Next Generation Sequencing Results}

MicroGen Diagnostics' comprehensive testing (patent pending) is a relative quantitative universal test for bacteria/fungi. DNA sequencing methods are used to identify the microorganisms' genetic signatures and the estimated percentage of organisms present in the specimen. Virtually all bacteria/fung are screened for and the most predominant populations are reported.

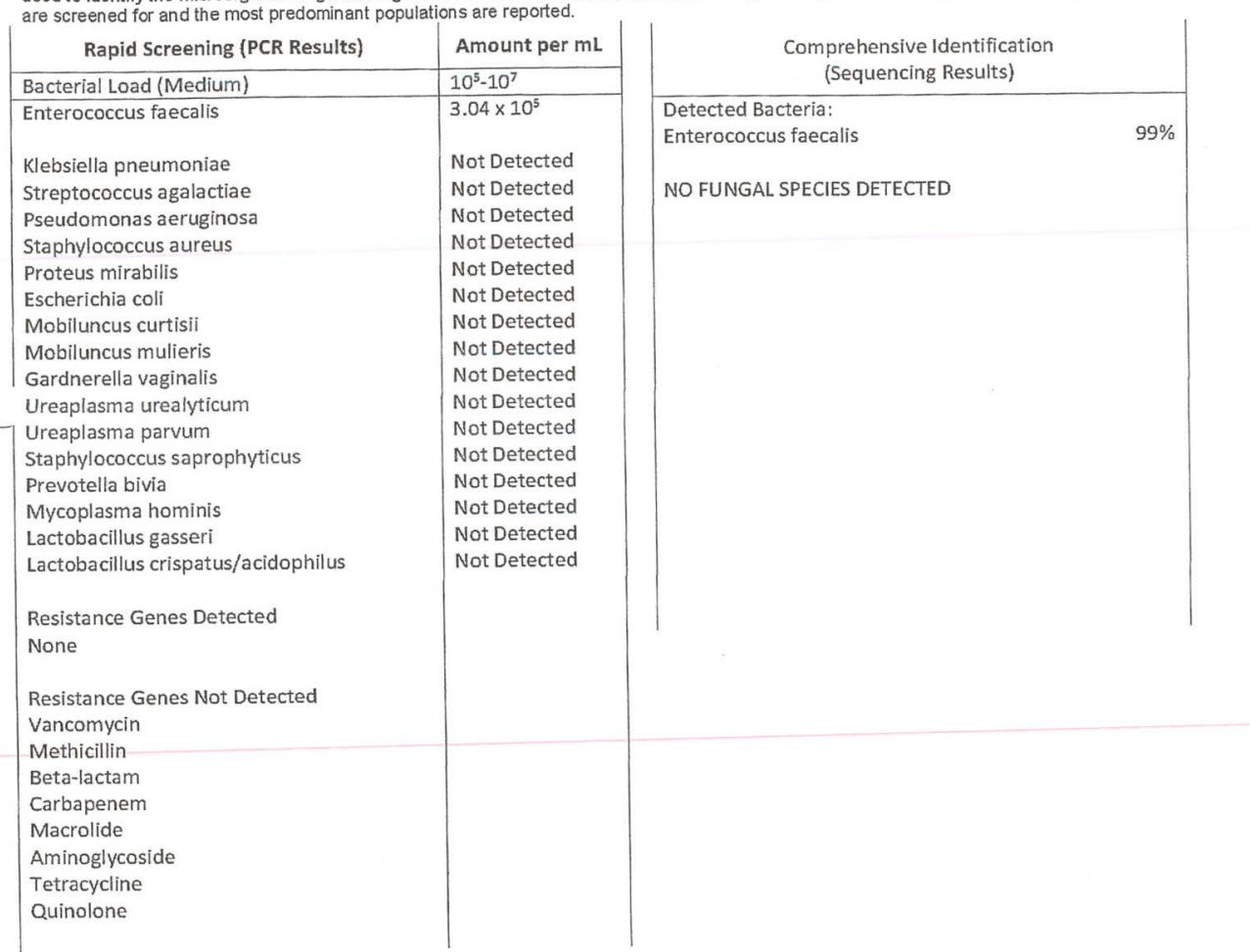

Complete Antibiotic Analysis [Next Page(s)]

ANTIEOTIC DISCL AIMER. Sounwest Regional PCR assumes no liability to patients with respect to the actions of physicians, health care facilities and other users, end is not respon sible for any injun

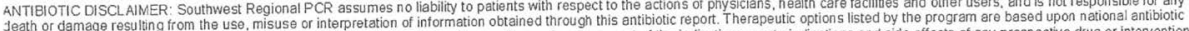

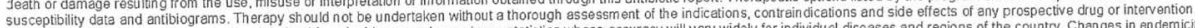
Furth ermore, the database is curated and derived from incidence and prevaience statistics whose accuracy will vary widely for individual diseases and regions of the country. Changes in end demichicy. incidence, and dings of cholce may occur. The list of dnugs. Inte ctious diseseses she
delay cannot be avoided. For more information please contact us at $855-208-0019$ 


\begin{tabular}{|c|c|c|c|c|c|c|c|}
\hline \multirow[b]{3}{*}{ Class } & \multirow[b]{3}{*}{ Generic } & \multirow{3}{*}{ 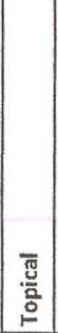 } & \multirow[b]{3}{*}{ 인 } & \multirow[b]{3}{*}{$\geq$} & & 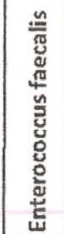 & 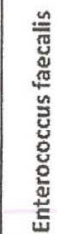 \\
\hline & & & & & Gram & + & + \\
\hline & & & & & Resp & FAn & FAn \\
\hline Aminoglycoside & amikacin & $\checkmark$ & & $\checkmark$ & & $\checkmark$ & $\checkmark$ \\
\hline $\begin{array}{l}\text { Extended spectrum penicillin/beta- } \\
\text { lactamase inhibitor }\end{array}$ & $\begin{array}{l}\text { amoxicillin/clavulanate } \\
\text { (Augmentin) }\end{array}$ & & $\checkmark$ & & & $\checkmark$ & $\checkmark$ \\
\hline fluoroquinolone & levofloxacin & & $\checkmark$ & $\checkmark$ & & $\sqrt{v}$ & $\sqrt{v}$ \\
\hline Glycopeptide & vancomycin & $\checkmark$ & & $\checkmark$ & & $\checkmark$ & $\checkmark$ \\
\hline & nitrofurantoin & & $\checkmark$ & & & $\checkmark$ & $\checkmark$ \\
\hline & tmp/smx (Bactrim) & $\checkmark$ & $v$ & $\sqrt{ }$ & & $\checkmark$ & $\checkmark$ \\
\hline Oxazolidine & linezolid & $v$ & $\checkmark$ & $\checkmark$ & & $\checkmark$ & $\checkmark$ \\
\hline penicillins & penicillin v & & $v$ & & & $\checkmark$ & $\checkmark$ \\
\hline $\begin{array}{l}\text { Gram Stain } \\
\text { +: Positive, -: Negative, I:Indetermina } \\
\text { Respiration } \\
\text { Ae:Aerobic, An: Anaerobic, FAn: Fac } \\
\text { * Resistance genes found. Consultati } \\
\text { recommendations made at the discr } \\
\text { concentrations is recommended. }\end{array}$ & $\begin{array}{l}\text { N: not applicable U: Unk } \\
\text { ative anaerobic, Unk: Unk } \\
\text { with a pharmacist on an } \\
\text { on of the physician based }\end{array}$ & $\begin{array}{l}\text { iate } \\
\text { Wn in }\end{array}$ & cour: & & $\begin{array}{l}\text { treat } \\
\text { n and }\end{array}$ & ent wi & \\
\hline
\end{tabular}

Supplemental Figure S2. Case scenario 2 with microgen. 
Case Scenario 3

A woman in her early $60^{\prime}$ s presents with a right ureteral stone and nephrolithiasis. She has no known allergies to antibiotics. Approximately 2 months prior, she developed a fever and chills and was diagnosed with a UTI. She was treated with antibiotics and her symptoms improved. The patient is now planned to undergo bilateral ureteroscopy. SOC urine culture result showed 'Multiple organisms present, each less than $10,000 \mathrm{CFU} / \mathrm{mL}$. These organisms, commonly found on external and internal genitalia, are considered to be colonizers. No further testing performed.'

What would you select as your antibiotic for surgery prophylaxis?

Based on Microgen results:

1. Would you change your antibiotic?

2. What would you select?

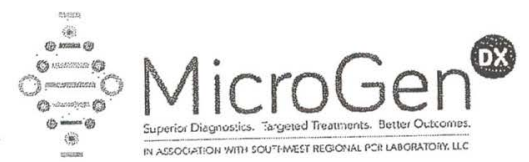

4321 Marsha Sharp Fwy Door 2, Lubbock, TX 79407

\section{Case Sunario 3}

Next Generation Sequencing Results

MicroGen Diagnostics' comprehensive testing (patent pending) is a relative quantitative universal test for bacteria/fungi. DNA sequencing methods are

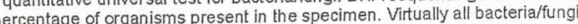
used to identify the microorganisms' genetic signatures and the estrmat

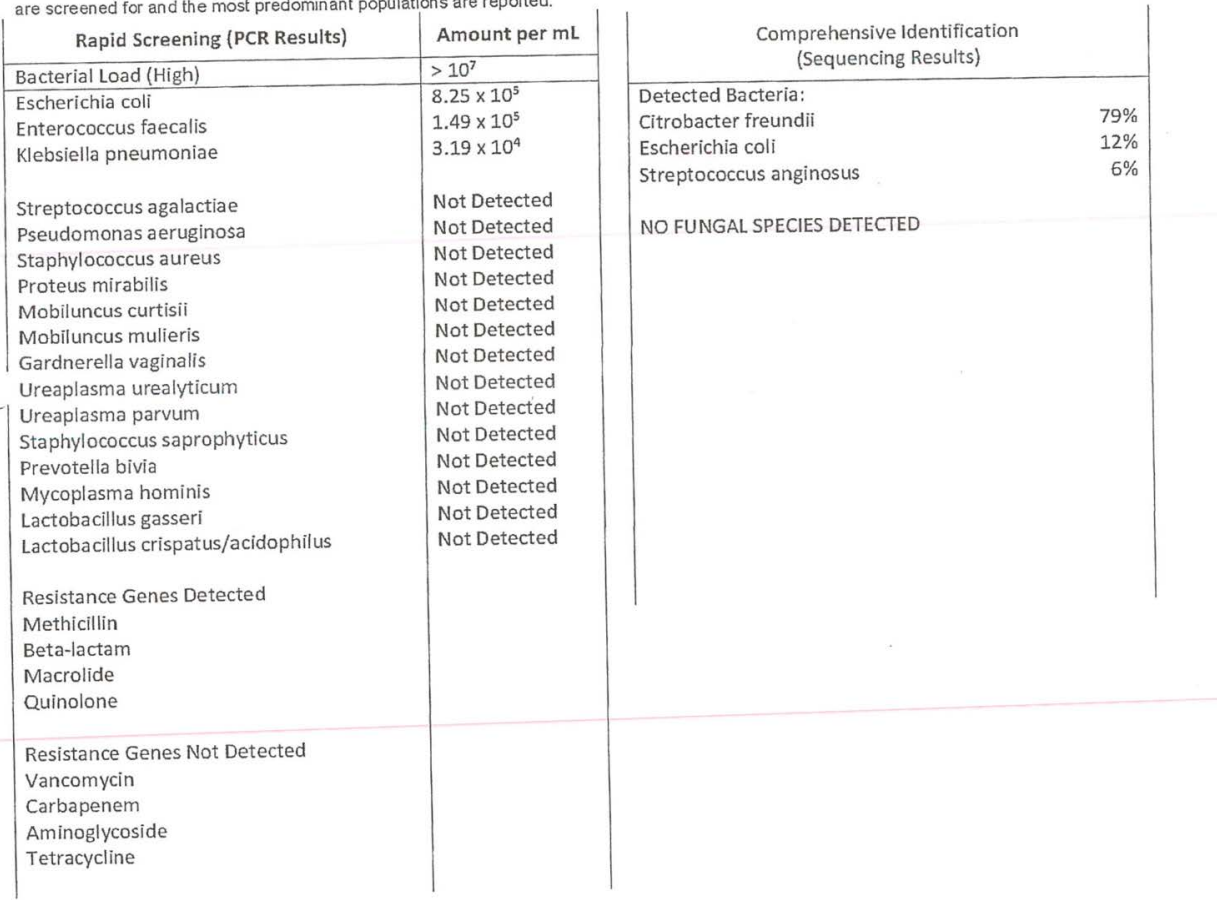

Complete Antibiotic Analysis [Next Page(s)]

is

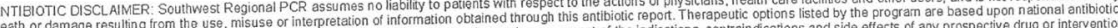

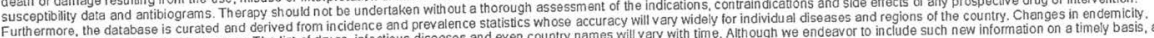
Furthermore, the database is curated and derived from incididence and prevalencect sat

delay cannot be avoided. For more information please contact us at 855-208-0019 


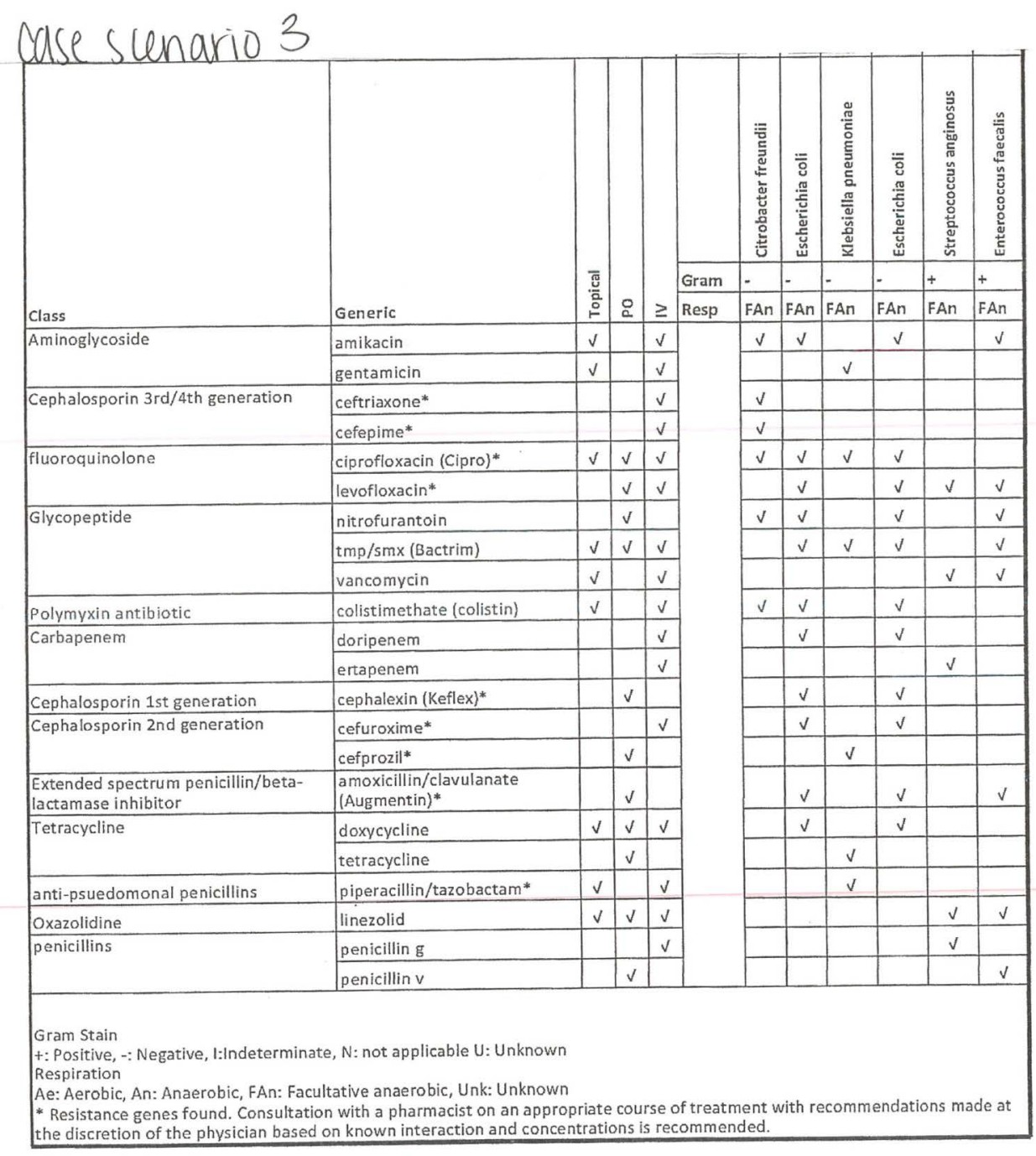

Supplemental Figure S3. Case scenario 3 with microgen. 